\title{
Perspectivas laborales de estudiantes de turismo en universidades de Puebla, México
}

\author{
Employment prospects of tourism students at universities \\ in Puebla, Mexico
}

\author{
María José Contreras-López \\ José Pedro Juárez-Sánchez y Benito Ramírez-Valverde
}

Colegio de Posgraduados. Campus Puebla, México

Resumen

En México, los egresados de la licenciatura en turismo enfrentan dificultades para obtener empleo. El objetivo de la investigación fue analizar la percepción de estudiantes de la licenciatura en turismo sobre su inserción al mercado laboral. En la investigación se utilizó el método mixto y se entrevistaron a estudiantes de turismo de tres universidades. Se encontró que los estudiantes perciben que están preparados y tienen altas expectativas en encontrar trabajo en pocos meses en áreas relacionadas con el turismo. Esperan devengar salarios regulares comparado con los que se ganan en el país, sí no encuentran empleo en lo que estudiaron estarían dispuestos a laborar en actividades diferente con la actividad turística. Se concluye que los estudiantes están preparados pero su incorporación al mercado laboral será difícil.

Palabras clave: Empleo, educación superior, turismo, ingresos, desigualdad.

Abstract

In Mexico, tourism graduates face difficulties in obtaining employment. The objective of the research was to analyze the perception of students of the degree in tourism about their insertion into the labor market. The research used the mixed method and tourism students from three universities were interviewed. It was found that students perceive that they are prepared and have high expectations in finding work in a few months in areas related to tourism. They expect to earn regular wages compared to those earned in the country, if they do not find a job in what they studied, they would be willing to work in activities other than tourism. It is concluded that students are prepared but their incorporation into the labor market will be difficult.

Key words: Employment, higher education, tourism, income, inequality. 


\section{INTRODUCCIÓN}

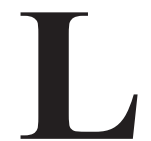

a crisis económica que enfrentan las economías nacionales se ve reflejada en el desempleo, donde uno de los sectores más afectados son las personas jóvenes y con mayor escolaridad. La Organización Internacional del Trabajo-OIT (2020) menciona que la tasa de desocupación en 2019 en América Latina fue de ocho por ciento, afectando más a las mujeres y a los jóvenes, siendo la tasa de desempleo tres veces superior a la tasa de desocupación de los adultos de 25 años o más, y representó 39.1 por ciento de los empleados (OIT, 2020). En México, a nivel general la tasa de ocupación en 2018 fue de 57.6 por ciento (CEPAL y OIT, 2019) y la tasa de desocupación urbana fue de 3.6 por ciento y en las personas jóvenes con educación superior fue de 14.5 por ciento (OCDE, 2019). Es de destacar que los egresados de mayor edad (35-44 años) poseen tasas de desempleo más bajas (tres por ciento) que los egresados jóvenes (5.7 por ciento) (OCDE, 2018). Después se ubican los trabajadores con estudios de nivel medio superior con una tasa de desempleo de cuatro por ciento y los que tienen educación básica se ubican en tres por ciento (OCDE, 2020).

Significa que hay una mayor demanda de mano de obra de menor calificación, y que los jóvenes universitarios tienen menores oportunidades de empleo como consecuencia de la crisis económica con sus respectivas repercusiones sociales y económicas. En este contexto, en las últimas décadas el turismo ha adquirido relevancia económica a escala mundial tanto en la llegada de turistas como por su aportación al Producto Interno Bruto (PIB) y generación de empleos, en 2017 alcanzó los 1,333 millones de turistas internacionales (Barometer, 2015) y en 2019 la llegada fue de 1,460 millones (World Tourism Organization-UNWTO, 2020), lo que significó un incremento de 9.5 por ciento. De acuerdo a la Organización Mundial del Turismo (OMT, 2021) en 2019, la actividad turística tuvo una derrama económica de 29.7 billones de pesos, participó con 10.4 por ciento del PIB mundial y generó 334 millones de empleos, es decir uno de cada cuatro de los nuevos puestos laborales en el mundo (World Travel and Tourism Council-WTTC, 2021) y sostiene uno de cada 11 puestos de trabajo en el mundo principalmente entre personas jóvenes.

En América Latina, en 2019 laboraba en el sector turístico diez por ciento de la Población Económicamente Activa (OIT, 2021) en donde las mujeres y los jóvenes conformaban 20.9 por ciento de los empleos generados y representaban 13.5 por ciento del empleo total (OIT, 2021). 
En 2019, México se ubicó en la séptima posición del Ranking del Turismo Internacional con 45 millones de llegadas (World Tourism Organization-UNWTO, 2020), generando 4'148,915 empleos directos en el periodo octubre-diciembre de 2020 y representaron, 8.5 por ciento del empleo nacional (SECTUR, 2021). Estos datos reflejan la importancia que el turismo ha adquirido en el contexto económico y ello ha influido en el incremento de la demanda de personal especializado. Es por lo que en América Latina las universidades públicas y privadas respondieron aumentando la oferta educativa con 906 instituciones educativas que ofertan programas para la instrucción del turismo, de las cuales 643 (71 por ciento) son privadas y 263 públicas. Destacando en América del sur: Brasil, Argentina, Venezuela y Perú (OMT, 2019).

En México, ante el crecimiento de la demanda de profesionistas especializados en las actividades turísticas, las instituciones de educación superior respondieron al llamado del mercado laboral. De acuerdo al Anuario Educación Superior (Licenciatura 2013-2014) que publica la Asociación Nacional de Universidades e Instituciones de Educación Superior (ANUIES, 2014) existían 2,718 universidades, con una oferta de 17,027 licenciaturas, de estas, 383 (2.2 por ciento) estaban relacionadas con la actividad turística como: Administración Turística, Gestión Turística, Desarrollo Turístico, Turismo, Turismo y Hotelería, Turismo y Comercio, así como Gastronomía, fundamentalmente. Cinco años después, (2018-2019) aumentó el número de universidades $(3,459)$ y su oferta educativa con 23,353 licenciaturas, de estas, 1,150 (4.9 por ciento) estaban relacionadas con la actividad turística con una matrícula de 108,162 estudiantes, que representan, 2.5 por ciento del total de estudiantes en el país. En el Estado de Puebla existen 124 licenciaturas relacionadas con la actividad turística (ANUIES, 2019).

Se puede decir que el aumento de la oferta educativa es una respuesta a la demanda del sector turístico, pero se considera que el equilibrio del mercado laboral no solo depende de la relación que existe entre el sector turístico y las universidades. También depende directamente del consumo de bienes y servicios proporcionados (auge económico), así como del precio de la mano de obra en relación con otros factores de la producción y de la posible mezcla técnica de factores de producción para proporcionar bienes y servicios, es decir, del progreso tecnológico que permite aumentos de la productividad en la industria y por lo tanto cae la proporción del trabajo empleado por cada unidad producida (Tribe, 1999). 
En este sentido, la licenciatura en turismo tiene una tasa de desempleo de 5.6 por ciento, tomando en cuenta que pertenece al campo de la administración y gestión de empresas de acuerdo a la clasificación de programas de estudio elaborada por la Secretaría de Educación Pública-SEP y el Consejo Nacional de Ciencia y Tecnología-CONACYT (IMCO, 2021). Esto señala que en esta carrera el número de desempleados es bajo en comparación con la licenciatura de Ciencias políticas (14.6 por ciento) Minería y extracción (10.9 por ciento), Ingeniería química (9.6 por ciento) y Diseño (9.3 por ciento). Esta profesión se ubica en el décimo noveno lugar (IMCO, 2021), siendo una licenciatura demandada laboralmente, pero no ocupaba los primeros lugares. Se puede decir que los egresados de la licenciatura en turismo uno de los problemas a la que estarán sujetos son las dificultades para encontrar empleo y cuando lo encuentran, este no está entre los mejor remunerados. El objetivo de la investigación fue analizar la percepción sobre inserción al mercado laboral turístico de los estudiantes de la licenciatura en administración turística de diferentes universidades del estado de Puebla.

\section{DESEMPLEO E INSERCIÓN LABORAL DE LOS ESTUDIANTES DE LICENCIATURA}

Se puede decir que el desempleo de los jóvenes universitarios no es nuevo, y sus causas son multifactoriales, solo que en las últimas décadas se considera que está relacionada con el modelo neoliberal que promueve la flexibilización del mercado laboral, para responder a las nuevas necesidades de acumulación de capital. Los neokeynesianos argumentan que la demanda efectiva determina el grado de empleo al incidir en las decisiones de los empresarios sobre el nivel de su producción futura, y está influirá en una mayor o menor contratación de empleados al decidirse sobre la utilización de la capacidad productiva existente de su empresa que implica acelerar o posponer la acumulación de capital (García y Cruz, 2017). Pero lo cierto es que la mano de obra no calificada juega un papel importante en la regulación de los costos laborales a escala mundial, constituyéndose el desempleo en una importante vía global de acumulación de capital al regular los costos laborales en cada una de las economías nacionales (Chossudovsky, 2007). Además, se debe tener en cuenta que las tasas de desocupación se relacionan fundamentalmente con el cambio tecnológico (Morales y Castro, 2015).

Una consecuencia de la acumulación de capital es el distanciamiento entre el trabajo y los derechos laborales, actualmente los contratos por 
tiempo indefinido y las prestaciones laborales son casi inexistentes entre los jóvenes que trabajan. Ante la tendencia que se observa en el mercado laboral y su respectiva flexibilidad, hoy, no solo se debe tener en cuenta la preparación personal de los estudiantes, debido a que el haber culminado o tener una licenciatura no asegura que el egresado tenga empleo y menos que este sea de calidad (Domínguez, 2017). En parte se explica a que la demanda de personas con estudios universitarios creció a una mayor tasa que la oferta de puestos de trabajo, lo cual conduce a un desajuste educacional entre los trabajadores y los puestos de trabajo que ocupan (Sánchez y Fernández, 2014).

Esta situación dio origen a la sobre-educación; que significa que el poseer un nivel educativo que supera las necesidades del puesto de trabajo conduce a tener un empleo, que era regularmente desempeñado por personas con un nivel de educación inferior y por un salario menor. Es por ello que, en términos de experiencia laboral, 85 por ciento de los egresados universitarios en México han tenido un trabajo remunerado (Centro de Opinión Pública de Laureate, 2017), 50 por ciento trabaja en áreas que no estudió y las empresas en 52 por ciento aseguran no encontrar el talento adecuado para cubrir sus vacantes (Expansión, 2020). Esto significa que solo una pequeña parte de los egresados de educación superior encontrará empleo asociado con la licenciatura estudiada, y que la gran mayoría tiene que aceptar puestos que en el pasado eran ocupados por personas con educación media.

Entonces, la sobre-educación puede entenderse como un fenómeno social que afecta la posición social de los individuos y desafía el papel de la educación como mecanismo de movilidad social y es una nueva forma de estratificación del mercado laboral, que coloca a los trabajadores sobre-educados en una posición de desventaja en relación con las individuos con el mismo nivel educativo, pero que están empleados en un puesto de trabajo adecuado (Capsada, 2017) cuando deberían tener la misma posición en el mercado laboral. Pero también, se refiere a los egresados de educación superior que se emplean en puestos en donde no se requieren formalmente estudios de licenciatura, ya que desarrollaran actividades en las que no emplean las competencias profesionales y transversales adquiridas, es decir que el empleo adquirido está por debajo del nivel educativo del trabajador (Murillo y Montaño, 2018).

Esto lleva a que ocupen un trabajo que anteriormente lo desempeñaban personas con menor nivel de enseñanza. También significa que los poseedores de un título de licenciatura obtendrán un trabajo de menor calificación 
para el que fueron preparados. Esto conduce a un desajuste ocupacional y a un malestar entre los estudiantes; ya que los universitarios que invirtieron recursos y tiempo en recibir una educación estarán insatisfechos, al no alcanzar un puesto acorde con su nivel educacional. Por otra parte, existe un grado de satisfacción superior, en el caso de los trabajadores que no estudiaron, pero que tienen un puesto donde normalmente se sitúan trabajadores con mayor formación (Sánchez y Fernández, 2014). Se puede decir que se produce una sobre-educación cuando el esfuerzo educativo no recibe la suficiente compensación económica y social en el mercado laboral o que el trabajo desarrollado requiere menores competencias para lo que fue preparado el estudiante universitario.

El fenómeno de la sobre-educación es uno de los principales indicadores que tienen en cuenta los empresarios al seleccionar o contratar personal. Significa, que la escolaridad entra en una espiral de crecimiento que no parece tener fin y repercute en un desequilibrio en el mercado laboral, ya que la oferta de empleo crece mucho más despacio (Gobernado, 2007). Bajo una visión economicista se debe buscar equilibrio entre la oferta y la demanda de licenciados en turismo, debido a que al tener una escasa formación de recursos humanos los empleadores reaccionan elevando los salarios y al inundar con más personal calificado el mercado, los empleadores disminuyen los salarios y suben en extremo los requisitos de acceso al empleo. En este sentido, Rahona (2008) menciona que un incremento en el nivel educativo beneficiaría al empleador, que contrataría a personas más cualificados a un salario menor, tal y como se observa en la actualidad.

Además, influye en el desempleo de los universitarios la distribución desigual de la matrícula, debido a que su concentración propicia el desequilibrio del mercado laboral por especialidad o disciplina. A este fenómeno lleva a la desprofesionalización de la educación superior; ya que los aumentos de la matrícula ocasionan que exista un desequilibrio de la oferta de egresados con respecto a los puestos de trabajo, se considera como un fenómeno recurrente de la masificación de la educación. En donde la ley general de equilibrio es la que determina el nivel de salarios que se debe pagar (Hernández y Salgado, 2014). Otro elemento a considerar es la vinculación universidad-empresa, sobre lo que Linarez (2014) argumenta que es una práctica común que en el sistema educativo las universidades elaboren planes y programas de estudio para contribuir a mejorar las condiciones económicas y personales de los estudiantes, pero también, se deben de buscar soluciones a los nuevos retos sociales y construir nuevos conocimientos. 
Es por esto que los organismos nacionales e internacionales se preocupan por el desarrollo de una política de calidad que contemple la práctica con la teoría, debido a que los egresados no están capacitados ni tienen los conocimientos que buscan las empresas. Dentro de la formación turística, se tiene una evidente crisis debido a que las demandas de este sector dieron origen al fracaso de los programas educativos, ya que hoy, se privilegian las competencias basadas en el conocimiento científico y tecnológico, así como las habilidades ligadas a su entendimiento y manejo (Valenti y Becerril, 2007). Ante ello, la estructura del sistema educativo y los currículos tienen un papel decisivo sobre el futuro proceso de transición hacia el mercado laboral.

De acuerdo a la Agencia Nacional de Evaluación de la Calidad y Acreditación (ANECA, 2009), el acceso del estudiante a un trabajo está regulado por su capacidad de formación en el puesto de trabajo (currículo educativo), y en algunos casos reflejan problemas en la calidad de la educación, lo que lleva a los empresarios a perder credibilidad sobre los títulos universitarios. Ello lleva a pensar que existe un desequilibrio entre la formación universitaria y lo que perciben los empleadores sobre su formación para el trabajo. También se debe tener en cuenta que entre las empresas adquiere relevancia el capital intelectual, ya que anteriormente solo daban importancia o hacían referencia a todas aquellas destrezas, habilidades y conocimientos de una persona que le permiten desempeñarse eficientemente en su puesto de trabajo, y hoy toman relevancia elementos como: la información, la propiedad intelectual o patentes, las bases de datos, los software, las marcas, que son consideradas como capital estructural y que por lo general pertenece a la institución. Es decir, que hoy prevale el capital humano que sumado al capital estructural da por resultado el capital intelectual (Villalobos et al., 2009) afectando la generación de empleos. Además, se debe de tener presente el impacto que la revolución tecnológica en la estructura del mercado de trabajo (Rodríguez y Moreno, 2017).

Es por ello que el sistema educativo universitario debe ser visto como punto importante dentro de nuestra vida y como una herramienta eficaz que favorezca el desarrollo. En donde la educación debe estar vinculada a los problemas y necesidades de cada región, debe formar cuadros profesionales con capacidad intercultural, con habilidades y destrezas para ser aplicadas en el ámbito laboral globalizado; con capacidad para adquirir lógicas productivas internacionales para trabajar en donde se les solicite (Aguilar, Campos y Sánchez, 2013). En un mundo global las empresas buscan a profesionistas con educación de calidad, es por eso que en los sistemas 
de enseñanza de alto rendimiento abarcan una amplia gama de modelos institucionales que en conjunto producen la variedad de trabajadores y empleados calificados que necesita el mercado laboral (Salmi, 2009).

Pero se observa en el nivel universitario que la preparación de los estudiantes no es de calidad y se refleja en los problemas que atraviesan cuando acceden al mercado laboral (Regalado y Oré, 2009). Según, Benavides (2004) tienen mejores ingresos y mayor reconocimiento los egresados de las universidades privadas que los que estudian en universidades públicas. Pero en la calidad de la enseñanza, existe una competencia desleal de las instituciones con bajos niveles de calidad que desprestigian al sistema universitario privado (Silas, 2005); y su proliferación afecta su futuro empleo. Entonces, se puede hablar de universidades de élite y universidades de menor calidad. Ello, es propiciado por el Estado con políticas de desregulación y reorientación de las universidades hacia el mercado que fomenta que las universidades privadas participen cada vez más en la matrícula total.

\section{Metodología}

En la investigación fueron seleccionadas tres universidades: dos públicas y una privada por la orientación con la que fueron creadas y las necesidades particulares que satisfacen, lo que las lleva a formar profesionistas para ocupar distintos puestos laborales. También obedece a que cuentan con prestigio institucional y poseen la mayor experiencia en la enseñanza en turismo, ya sea de negocios o alternativo a nivel licenciatura. De manera específica, la Universidad Madero (UMAD) fue elegida por su calidad académica al estar entre las instituciones privadas más importantes y con mayor trayectoria en la enseñanza de turismo en la entidad; la Benemérita Universidad Autónoma de Puebla (BUAP), se escogió por ser el claustro público más importante del Estado y una de las más destacadas del país, así como su experiencia en la enseñanza de esta materia y la Universidad Intercultural del Estado de Puebla (UIEP) por ser la única universidad intercultural en el Estado que imparte la licenciatura en turismo.

En 2019, la UMAD tenía una matrícula de 1,825 alumnos distribuidos en 27 licenciaturas, la escuela de Administración Turística contaba con 54 alumnos inscritos y su programa está integrado por nueve semestres para la obtención del título universitario. Tiene dos especialidades: Turismo de Negocios y Turismo Recreativo. Cuentan con un departamento de idiomas $\mathrm{y}$ de cómputo; y aseguran formar profesionales con un perfil gerencial y de liderazgo para desempeñarse en empresas, consorcios y organizaciones 
vinculadas a la actividad turística. Proporciona al estudiante competencias en el dominio del inglés, francés y alemán. El importe por estudiar en esta universidad tomando en consideración la cuota de admisión, y el pago de los créditos por cursar la licenciatura asciende aproximadamente a $\$ 420,355$ (UMAD, 2021). Se ubica en la zona conurbada de la ciudad de Puebla, específicamente en el municipio de San Andrés Cholula que detenta una población de 137,290 personas, 3.3 por ciento habla alguna lengua indígena, 37.6 por ciento tiene instrucción básica y 39.7 por ciento posee estudios de nivel superior (INEGI, 2016).

En 2019, la BUAP tenía una de las matriculas — 78,232 estudiantesmás grandes de México distribuidas en 100 licenciaturas. La carrera en Administración Turística tenía 1,978 alumnos. En su modelo educativo se maneja un sistema por créditos, se paga al inicio de cada curso una inscripción que va de los 764 a los 3,279.8 pesos y el costo de una carrera puede oscilar entre los 2,716.3 y cinco mil pesos. Sus egresados son formados en conocimientos, habilidades, actitudes y valores; y se les prepara en el idioma inglés. Al finalizar la licenciatura podrán incorporarse a laborar en complejos turísticos, en la industria hotelera, restaurantera, bares, agencias de viajes, transportadoras turísticas, guía de turistas, animador turístico, líneas aéreas, centros de convenciones; en la Administración Pública y en todas las actividades que conforman al sector turístico (BUAP, 2021). Tiene un laboratorio de gastronomía y un departamento de idiomas y computo, así como biblioteca. La BUAP se localiza básicamente en el municipio de Puebla, su población asciende a 1'576,259 personas, 3.1 por ciento de sus habitantes de 15 años o más es analfabeta y cuatro por ciento de las personas de tres años y más hablan alguna lengua indígena (INEGI, 2016).

Otro tipo de universidad en México la constituyen las interculturales que reconocen el derecho relativo a una educación diferenciada que hace referencia a la dimensión cultural del proceso educativo y ha sido bandera clave para dotar de carácter decolonial y liberación, mediante nuevas apuestas educativas que abandonan la educación convencional u occidental para contribuir a disminuir la desigualdad económica y social (Vargas, 2014). De acuerdo a Mateos y Dietz (2016) su oferta educativa y currículum es flexible caracterizándose porque no trata de ampliar la cobertura, sino de redefinir el modelo de universidad, adaptando las carreras a los contextos rurales e indígenas, ofreciendo una formación que combina excelencia académica con pertinencia cultural y lingüística; que no inunde el mercado laboral de la región y que los forme en competencias para el autoempleo. 


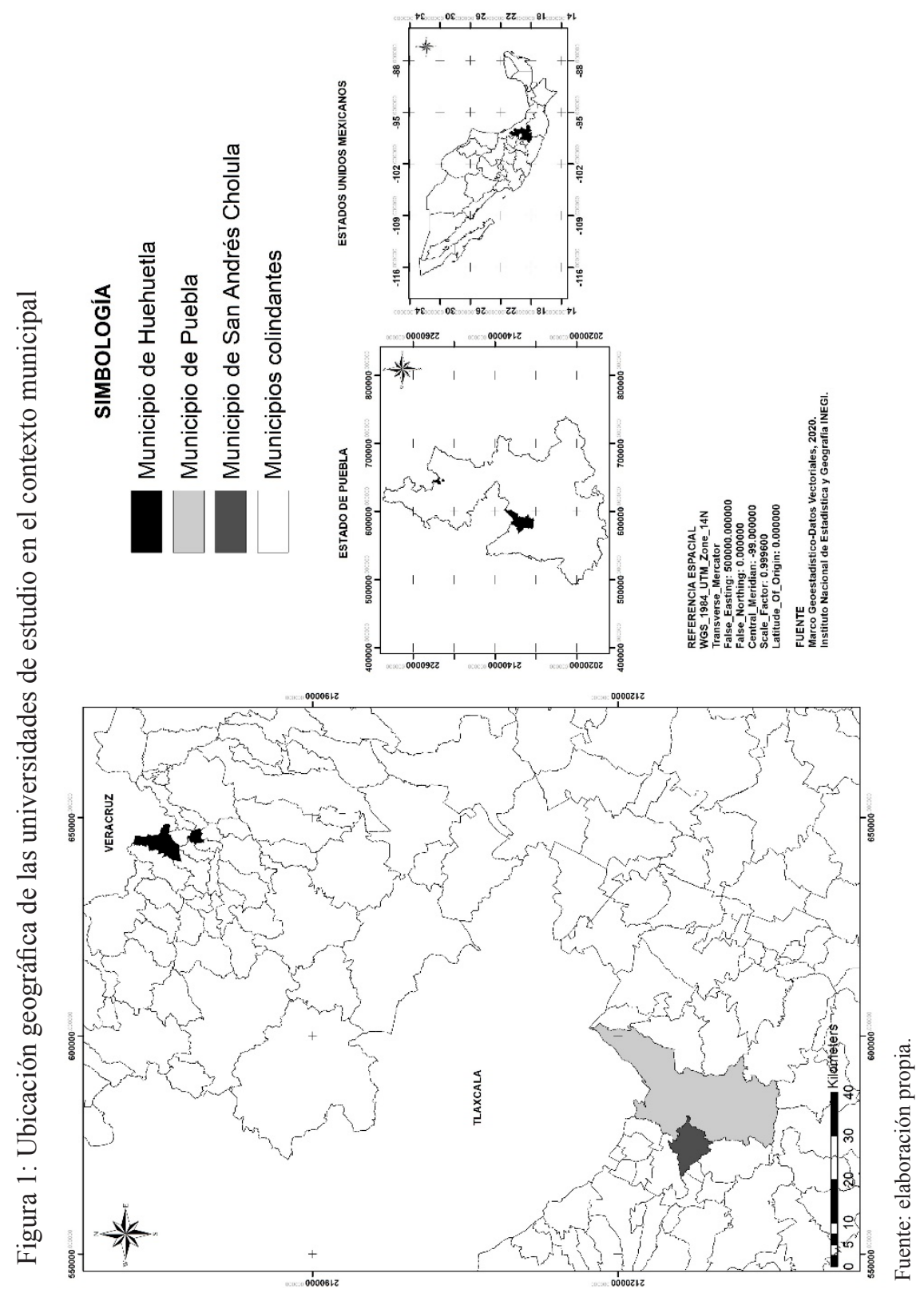


Esta opción, representa para la población predominantemente indígena ubicada en regiones poco accesibles, una oportunidad para ingresar a la universidad, ya que la gran mayoría de los estudiantes de estas universidades, difícilmente lograrían inscribirse a una universidad convencional.

La UIEP es una universidad pequeña y de reciente creación, en 2019 tenía 855 estudiantes distribuidos en seis licenciaturas, su oferta educativa está relacionada directamente con el desarrollo de las comunidades de la región de la Sierra Nororiente de Puebla. En ella se forman jóvenes con una sólida vinculación comunitaria fortaleciendo la lengua y la cultura originaria con el objetivo de formar profesionales capaces de construir alternativas de desarrollo turístico (UIEP, S/F). El 81 por ciento del profesorado cuenta con posgrado y la Licenciatura de Turismo Alternativo tiene 107 alumnos. Al egresar los estudiantes adquieren competencias para ser especialistas en turismo en el ámbito regional, ya sea a través de la creación de empresas o la gestión de proyectos turísticos, así como emplearse en el sector turístico local. La universidad se ubica en el municipio de Huehuetla, que tiene una población de 18,803 personas, 27.9 por ciento de la población de 15 años o más no tiene instrucción y el 56.8 por ciento estudio la primaria. EL 89.7 por ciento de las personas de tres años o más hablan alguna lengua indígena (INEGI, 2016) y 86.4 por ciento de su población está en pobreza.

El estudio fue de corte transversal y comparativo, en la investigación se utilizó el método mixto (Hernández et al., 2014) e inició con una revisión bibliográfica. Para recolectar la información se basó en la técnica del cuestionario y la observación directa, significa que el investigador estuvo en el lugar donde se desarrolló el trabajo de campo (Mendoza, 1994). El cuestionario se estructuró en las siguientes partes: i) Características generales de los entrevistados (Edad, sexo, residencia, lugar de estudio, entre otros), ii) Competencias profesionales (Idiomas, Adquisición de Conocimientos del Área de Turismo, Trabajo en Proyectos, Gestión Empresarial), iii) Competencias transversales (Trabajo en Equipo y Tecnologías de la Información y Comunicación) y iv) Inserción laboral (Expectativas de trabajo, tiempo que invertirían y lugar en donde laborara, ingresos, capacitación. La mayoría de los ítems fueron cerrados y la entrevista se realizó cara a cara entre el entrevistador y el entrevistado.

El cuestionario fue aplicado a estudiantes de turismo de las tres universidades, encontrándose como problema la diferencia en el tamaño de estas y en el número de estudiantes de la licenciatura en turismo, ante ello se diseñó un esquema de muestreo diferente en cada universidad. Es por 
lo que se decidió tomar a todos los estudiantes de turismo de la UMAD y UIEP como población en estudio. En el caso de la BUAP, la Universidad de mayor tamaño, se planteó el uso de un muestreo estadístico donde el marco de muestreo consideró a todos los estudiantes $(1,300)$ de la escuela de administración turística. Se consideró la variable de posibilidad de obtener un empleo en el área de turismo al concluir sus estudios, por lo que se decidió por un muestreo cualitativo. Se analizó la posibilidad de utilizar un muestreo estratificado por semestre de estudio en la Universidad, situación posible en la BUAP, pero por el reducido tamaño de la población de las otras universidades no se podría realizar una estratificación adecuada para la comparación entre estratos y entre universidades. La ecuación 1 para estimar el tamaño de la muestra, es presentada por Gómez (1979) y se especifica de la siguiente forma:

$$
\mathbf{n}=\frac{\mathrm{N} Z^{2}{ }_{\alpha / 2} p_{n} q_{n}}{\mathrm{~N} \mathrm{~d}^{2}+Z^{2}{ }_{\alpha / 2} p_{n} q_{n}}
$$

donde:

$\mathrm{N}=$ Tamaño de población

$\mathrm{z}_{\alpha / 2}=$ Confiabilidad

$\mathrm{d}=$ Precisión

$\mathrm{p}_{\mathrm{n}}=$ Proporción con la característica de interés

$\mathrm{q}_{\mathrm{n}}=$ Proporción sin la característica de interés

La población estuvo compuesta por 1,300 estudiantes (ANUIES, 2014) y una confiabilidad del 95 por ciento $\left(Z_{\alpha / 2}=1.96\right)$. Considerando la varianza máxima y para definir el tamaño de muestra se recurrió a la variable: al egresar de la licenciatura considera que puede encontrar trabajo en el área de turismo $\left(\mathrm{p}_{\mathrm{n}}=0.5 \mathrm{y} \mathrm{q}_{\mathrm{n}}=0.5\right)$. Sustituyendo los valores en la ecuación y agregando diez por ciento de seguridad, el tamaño de la muestra fue de 64 estudiantes que se encontraban en la fase final de su licenciatura y fueron seleccionados aleatoriamente. En la UMAD se realizó el mayor número de entrevista - 32- de acuerdo al Anuario Educación Superior - Licenciatura 2013 - 2014 de ANUIES (2014), la universidad tenían registrados en la licenciatura un total de 41 alumnos. En la UIEP se realizó un censo, es decir se entrevistó a 15 personas que representan la totalidad de alumnos de turismo alternativo de la Universidad (ANUIES, 2014). Para analizar la información en primer lugar se trabajó en la revisión de los cuestionarios, 
con ellos se construyó una base datos en Excel y posteriormente se editó la información capturada en esta. Posteriormente se trasladó la base de datos a un programa estadístico y para la interpretación de los resultados se conformaron tres grupos de acuerdo con las universidades estudiadas - UMAD, BUAP y UIEP-.

En la interpretar de los resultados, de acuerdo con la escala de medición de la variable analizada, se recurrió a la aplicación de la prueba estadística de tipo no paramétricas y paramétricas. En el caso de la comparación de las tres universidades, las pruebas no paramétricas utilizadas fueron Kruskal-Wallis y en el caso de las pruebas estadísticas cuantitativas se empleó el análisis de varianza (Anova) con un criterio de clasificación. También se recurrió a la prueba de la $t$ de Student (variables cuantitativas) y de Chi cuadrada $\left(\chi^{2}\right)$ (variables cualitativas) para estudiar dos grupos. Se utilizó la regresión logística con el método de selección por pasos hacia adelante (Wald). La regresión logística fue aplicada en el análisis de datos derivados de condiciones experimentales, recientemente, estos modelos contemplan datos obtenidos en estudios observacionales (Pregibon, 1981). Es el caso de la información que se trabaja en esta investigación. El modelo de regresión logística, es utilizado cuando la variable respuesta es discreta (Hosmer, Lemeshow, y Sturdivant, 2013). En este estudio se quiere conocer las variables relacionadas con la competitividad laboral de los estudiantes de turismo. Entonces se tiene una variable respuesta binomial y se requiere conocer con que variables se asocian. La regresión logística es buena alternativa y el modelo sería:

$$
\ln (\pi / 1-\pi)=\beta_{0}+\beta_{1} x_{1}+\cdots+\beta_{p} x_{p}
$$

Donde la variable respuesta estará en función de $\beta_{0}, \beta_{1}, \ldots \beta_{\mathrm{p}}$ son parámetros, desconocidos y $\mathrm{X}_{1}, \mathrm{X}_{2} \ldots \mathrm{X}_{\mathrm{p}}$ son variables explicatorias.

\section{Perspectivas laborales de los estudiantes DE UMAD, BUAP Y UIEP}

Las personas que estudian este tipo de licenciatura son jóvenes con edad promedio de 20.9 años, estadísticamente, mediante un análisis de varianza $(\mathrm{F}=1.139 ; \mathrm{p}=0.256)$, se encontró que la edad fue similar entre los estudiantes de la UMAD (21.2 años), BUAP (20.7 años) y UIEP (21 años), significa que los alumnos se encuentran en la edad escolar que les corresponde y es especialmente notable el caso de los que estudian en la Universidad 
Intercultural, en donde los alumnos por lo regular tienen problemas para continuar sus estudios, debido a que no tienen los recursos económicos para financiar su educación. Con referencia a su estado civil se encontró que 96.7 por ciento están solteros y la mayor parte ( 77 por ciento) de los alumnos de esta licenciatura son mujeres. Este resultado se considera alto, comparado con el porcentaje (39.6 por ciento) de mujeres que egresaron de las licenciaturas en ingeniería de las Universidades Tecnológicas del país (Presidencia de la República, 2015) y es muy similar (81 por ciento) al estudio que realizó Ramírez (2018) a los egresados de la licenciatura en turismo del Centro Universitario en Ciencias Administrativas de la Universidad de Guadalajara. Se considera que la profesionalización de la mujer en el sector turístico permitirá su acenso a empleos con mayor responsabilidad, y se abandone la idea de que los trabajos que desempeñan las mujeres en el sector turístico no son más que la prolongación del trabajo doméstico y de los roles tradicionales que desempeñan en el hogar (Mendoza y Chapulín, 2015).

Sobre la formación de los estudiantes, 59.8 por ciento egresó de preparatorias públicas, es de resaltar que 79.4 por ciento de los egresados de preparatorias privadas continuaron sus estudios en instituciones particulares, fue el caso de la UMAD. En la BUAP, 69.9 por ciento y en la UIEP la totalidad de sus alumnos estudiaron en escuelas públicas, ya que, para ambos segmentos, es demasiado costoso lo que para la mayoría hace inaccesible asistir a una escuela privada debido a la situación económica de sus familiares y en el caso particular de los estudiantes de la UIEP, provienen de familias que viven en condiciones de muy alta marginación y pobreza, y no tienen otra oferta educativa. Los porcentajes obtenidos en la UMAD y BUAP respecto a la procedencia académica de sus estudiantes se consideran bajos, al compararlos con la matrícula (81 por ciento) estudiantil del nivel medio superior que concurre a escuelas públicas en México (Secretaría de Educación Pública-SEP, 2020). Debe señalarse que las mujeres (41.5 por ciento) estudiaron un poco más en escuelas privadas que los hombres (35.7 por ciento).

La razón por la que los entrevistados decidieron estudiar esta licenciatura fueron diversas: el emplearse en el sector turístico (45.9 por ciento), porqué encontraron en ella su vocación (17.2 por ciento), el mismo porcentaje externó que la carrera tiene mucha demanda de trabajo, en menor porcentaje citaron que pueden laborar donde viven (5.7 por ciento), por los salarios que devengarían ( 3.3 por ciento) y el prestigio de la carrera (4.1 por ciento), fundamentalmente. En la UMAD, mencionaron que es en 
donde les agradaría ocuparse (64.7 por ciento), que existe demanda laboral (17.6 por ciento) y señalaron que es su vocación ( 8.8 por ciento). En la BUAP, se obtienen resultados similares a los de la UMAD, indicaron que es donde quieren desempeñarse (46.6 por ciento), que es su vocación (21.9 por ciento) y que existen empleos en el sector (13.7 por ciento). En la UIEP se obtiene resultados un poco distintos, 33.3 por ciento mencionó que le da la oportunidad de emplearse en donde viven, otro porcentaje similar argumentó que hay trabajo en el sector turístico y que existen buenos salarios (20 por ciento) esencialmente. Por sexo, estadísticamente $\left(\chi^{2}=3.921 ; \mathrm{p}<0.048\right)$ se obtuvieron resultados diferentes, en las mujeres destacó que es donde les gustaría trabajar (48.9 por ciento), que es su vocación (17 por ciento) y que existe demanda de trabajo (48.9 por ciento). Los varones acentuaron que les agradaría desempeñarse en actividades turísticas ( 35.7 por ciento), que hay trabajo ( 25 por ciento) y que es su vocación (17.9 por ciento).

Estos resultados coinciden con los encontrados por Torres (2020) sobre las expectativas de los estudiantes en turismo de la Universidad de Almería, España, al argumentar que estudiaron esta licenciatura porque se sentían atraídos por trabajar en hoteles, agencias de viajes, oficinas de turismo, así como por aprender idiomas, por las salidas laborales de este grado y en menor medida por vocación. Es importante acentuar que 63.1 por ciento de los entrevistados estudiaron esta licenciatura debido a que les gustaría desenvolverse en actividades propias de la carrera, es decir, en realizar actividades que les agradan. En este contexto, Gómez y Arroyo (2015) mencionan que para que sean felices los jóvenes deben desarrollarse como personas y ello implica tener un trabajo que les guste. Ello repercute en que los empleados tengan una mayor productividad en el trabajo.

Pero, no solo es suficiente poseer un empleo agradable, también es transcendental preparar adecuadamente a los estudiantes para que afronten las necesidades del mercado de trabajo, en este escenario adquiere relevancia el desarrollo de las competencias profesionales, en donde los programas de estudio deben tener en cuenta las necesidades laborales y el contexto organizacional (Danvila et al., 2013) para capacitarlos y desempeñen sus actividades con eficiencia y eficacia. En esta investigación las competencias profesionales se agruparon en conocimientos técnicos y habilidades concretas, mientras que las competencias transversales reflejarían el modo en que una persona realiza su trabajo, ambas competencias vendrían a integrar conocimientos, habilidades, actitudes y valores para que los estudiantes logren desarrollar los mecanismos necesarios para contrarrestar 
apropiadamente las diversas situaciones e inconvenientes que surjan a lo largo de su vida personal y profesional (Sáenz et al., 2021).

Con respecto a las competencias profesionales que adquirieron, 85.2 por ciento comentó que hablan inglés, en la UMAD la totalidad lo habla y en menor proporción lo dominan en la BUAP (87.7 por ciento) y UIEP (40 por ciento). De estos, 63.8 por ciento afirmó hablarlo regular o bien, sobresaliendo la UMAD (32.4 por ciento) y la BUAP (18.5 por ciento) al comentar que conversan muy bien este idioma. Los de la UIEP, 50 por ciento dijo que lo hablan bien y 33.3 por ciento regular. Aquí, las mujeres ( 88.3 por ciento) lo hablan más que los hombres ( 75 por ciento). Se encontró que muy pocos saben francés (34.4 por ciento), es de destacar que en la UMAD (73.5 por ciento) y en la BUAP (23.3 por ciento) manifestaron hablar esta lengua. En la UMAD, 66 por ciento lo parla entre muy bien y bien y 36 por ciento lo habla de manera regular, en la BUAP, lo hablan entre regular (76.5 por ciento) y bien o muy bien ( 23.5 por ciento). El 35.1 por ciento de las mujeres y 32.1 por ciento de los hombres hablan este idioma. En porcentaje muy bajos (21.3 por ciento) manifestaron tener conocimiento del idioma alemán. La UMAD (64.7 por ciento) se distinguió en este tipo de competencias, en la BUAP solo 5.5 por ciento dijo hablarlo. En la UMAD lo hablan entre muy bien y bien (31.8 por ciento) y de manera regular (59.1 por ciento), fundamentalmente. En este rubro se considera que hace falta fortalecer esta competencia y se tiene que hacer especial énfasis en dominar el idioma inglés (Rivas et al., 2019).

En la adquisición de conocimientos del Área de Turismo, 87.7 por ciento mencionó que adquirió esta competencia entre bien y muy bien, entre universidades estadísticamente (Kruskal-Wallis $=1.905 ; \mathrm{p}=0.386$ ) no existió diferencia en esta competencia. En la UMAD, 75.5 por ciento las desarrolló muy bien o bien y 23.5 por ciento de manera regular y en la BUAP, 83.2 por ciento las adquirió bien o muy bien, 5.5 por ciento de manera regular y 1.4 por ciento no las desarrollo. En la UIEP, 86.7 por ciento argumentó que se apropiaron muy bien o bien de esta competencia y 11.5 por ciento de forma regular. Con respecto a la competencia de Prestación de Servicios Turísticos, 73.8 por ciento la desarrolló entre bien y muy bien, aquí la BUAP (79.4 por ciento) destacó sobre la UMAD (66.6 por ciento) y la UIEP (60 por ciento) al apropiarse de esta competencia entre bien o muy bien. Las mujeres ( 73 por ciento) y lo hombres ( 75 por ciento) lograron resultados muy similares.

En la competencia de Trabajo en Proyectos los resultados se consideraron regularmente aceptables, ya que 68.9 por ciento la desarrollo muy 
bien o bien, en este sentido, los entrevistados de la UMAD (76.5 por ciento), BUAP (71.2 por ciento) y UIEP (40 por ciento) arguyeron que las desarrollaron muy bien o bien, a pesar de estos resultados no se encentró diferencia estadística (Kruskal-Wallis $=13.803 ; \mathrm{p}=0.087$ ) entre las universidades. En la Gestión Empresarial, se encontró diferencia estadística (Kruskal-Wallis $=23.648 ; \mathrm{p}=0.003$ ) debido a que los estudiantes de la UMAD (64.7 por ciento) y la BUAP (64.4 por ciento) se apropiaron mejor de esta competencia (bien o muy bien) y en la UIEP, 73.3 por ciento las desarrolló de forma regular. Con respecto a la competencia de Calidad, se encontraron resultados similares estadísticamente (Kruskal-Wallis $=4.832$; $\mathrm{p}=0.565)$ entre la UMAD (76.5 por ciento), BUAP (65.75 por ciento) y UIEP (66.7 por ciento) al mencionar que se apropiaron bien o muy bien de esta área del conocimiento.

En Atención al Cliente de manera general, 77.1 por ciento y de manera específica, en la UMAD (82.3 por ciento), la BUAP (78.1 por ciento) y la UIEP (60 por ciento) las adquirió bien o muy bien, estadísticamente no existió diferencia (Kruskal-Wallis $=11.832 ; \mathrm{p}=0.154)$ entre universidades. En la Realización de Eventos, tampoco se encontró diferencia estadística (Kruskal-Wallis $=8.147 ; \mathrm{p}=0.419$ ) a pesar que en la UMAD (73.5 por ciento) se observó que aprovecharon muy bien o bien este tipo de competencias en comparación con la BUAP (65.7 por ciento) y UIEP (53.4 por ciento). Los resultados contradicen la percepción que tiene sobre su formación profesional al encontrarse que no desarrollaron a plenitud sus competencias profesionales.

En las competencias transversales como el Trabajo en Equipo, 83.6 por ciento dijo que la desarrolló muy bien y bien, no se halló diferencia estadística (Kruskal-Wallis $=1.437 ; \mathrm{p}=0.487$ ) entre universidades. Es por lo que en la UMAD ( 88.3 por ciento), la BUAP (75 por ciento) y en la UIEP ( 66.7 por ciento) mencionaron que se apropiaron de esta competencia muy bien y bien. Fueron muy pocos (26.4 por ciento) los que las adquirieron de manera regular o mal. En el desarrollo de las Tecnologías de la Información y Comunicación, las catalogaron entre buenas y regulares $(75.4$ por ciento), solo 16.4 por ciento las consideró como muy buenas, no se encontró diferencia estadística (Kruskal-Wallis $=0.212 ; \mathrm{p}=0.899$ ) entre la UMAD al mencionar 14.7 por ciento que fueron muy buenas y 75.5 por ciento entre regulares y buenas, en la BUAP, 16.4 por ciento argumentó que fueron muy buenas y 54.8 por ciento dijo que fueron entre buenas y regulares. En la UIEP, 20 por ciento mencionó que este tipo de competencias fueron regulares ( 60 por ciento), buenas ( 20 por ciento) y muy buenas 
(20 por ciento). Los estudiantes de la BUAP y UMAD tienen una mejor precepción de las competencias que desarrollaron, mientras que los de la UIEP las adquirieron en menor proporción.

En términos generales, 87.7 por ciento consideró que están bien preparados para incorporarse al mercado de trabajo y que serán competitivos en su inserción laboral, estadísticamente (Kruskal-Wallis $=3.876 ; \mathrm{p}=0.423$ ) no existió diferencia entre la UIEP (93.3 por ciento), la UMAD (91.1 por ciento) y la BUAP ( 84.9 por ciento). Es de desatacar que las mujeres ( 90.4 por ciento) valoraron ser más competitivas que los hombres (78.6 por ciento). Al indagar los factores que se encuentran relacionados con la competitividad laboral y conocer que variables presentan mayor influencia (variable respuesta dicotómica) se realizó una regresión logística mediante el método de selección de variables por pasos hacia adelante (Wald), aplicado a un grupo de variables educativas, sociales y económicas. Se buscaron variables que tuvieron mayor relación con la competitividad laboral entre los estudiantes de las distintas universidades de estudio, y se procedió a seleccionar el modelo de regresión logística con la información obtenida en las encuestas aplicadas. Mediante, un procedimiento de selección de modelos se procedió a incluir y desechar variables, hasta obtener el modelo adecuado. Finalmente se encontró que la forma en que dan su clase los profesores en el área de conocimientos generales y los cursos de capacitación que toman los profesores en idiomas, fueron las variables que resultaron significativas (Tabla 1) al manifestar asociación con la competitividad laboral.

Tabla 1: Estimadores del modelo de regresión logística con el método de selección por pasos hacia adelante (Wald)

\begin{tabular}{lccccc}
\hline Variables & B & E.T. & Wald & P & $\operatorname{Exp}(\mathrm{B})$ \\
\hline $\begin{array}{l}\text { Forma impartir clase área } \\
\text { conocimientos generales }\end{array}$ & 0.500 & 0.203 & 6.079 & 0.014 & 0.607 \\
$\begin{array}{l}\text { Capacitación de profesores } \\
\text { del área de idiomas }\end{array}$ & 0.991 & 0.323 & 9.396 & 0.002 & 2.693 \\
\begin{tabular}{l} 
Constante \\
\hline
\end{tabular} & -1.115 & 1.795 & 0.386 & 0.535 & 0.328 \\
\hline
\end{tabular}

Fuente: elaboración propia a partir de datos de encuesta, 2015.

Por lo que se plantea que la competitividad tiene efectos directos e indirectos sobre la inserción laboral, en este sentido, los estudiantes consideraron relevante el papel de los profesores en la forma en como imparten sus cursos en el área de conocimientos generales. Esta variable fue calificada en una escala del 0 al 10 y en promedio obtuvieron los profesores 8.1 puntos, encontrándose diferencia estadística $(t=3.33 ; p=0.001)$ entre 
los estudiantes que se consideraron competitivos y no competitivos. Es importante mencionar que la forma que enseñan los profesores del Área de Idiomas obtuvo una calificación de 8.6 y se encontró diferencia estadística $(t=2.979 ; p=0.003)$ entre los estudiantes. En este rubro las otras áreas obtuvieron una menor calificación. También le dieron bastante peso a la capacitación, especialmente a los idiomas, en ese sentido, se encontró que fue una de las áreas en donde los estudiantes (41.8 por ciento) consideraron entre alta y muy alta la asistencia a cursos de capacitación por parte de sus profesores.

La mayoría (95.6 por ciento) de los estudiantes mencionó que tienen altas expectativas en encontrar trabajo en alguna área relacionada con el turismo. En la UIEP, la totalidad estudiantes consideran que al egresar obtendrán trabajo, 94.1 por ciento de la UMAD y 95.9 por ciento de la BUAP. Estos resultados son altos comparados a los obtenidos en la investigación realizada a los egresados de la licenciatura en turismo del Centro Universitario en Ciencias Administrativas de la Universidad de Guadalajara, en la que se menciona que 69 por ciento de sus egresados está laborando y de estos, 22.1 por ciento se encontraba trabajando en algo que no estaba relacionado con su formación profesional (Ramírez, 2018). Para conocer qué factores se encuentran relacionados con la expectativa de encontrar trabajo, se utilizó el modelo de regresión logística mediante el método de selección de variables por pasos hacia adelante (Wald) aplicado a un grupo de variables, en este análisis se encontró que lo más relevante para los estudiantes, son los temas de los cursos que se imparten en al área de idiomas (Tabla 2).

Tabla 2: Estimadores del modelo de regresión logística con el método de selección por pasos hacia adelante (Wald)

\begin{tabular}{lccccc}
\hline Variables & $\mathrm{B}$ & E.T. & Wald & $\mathrm{P}$ & $\operatorname{Exp}(\mathrm{B})$ \\
\hline Cursos en área idiomas & -0.857 & 0.272 & 9.949 & 0.002 & 0.425 \\
Constante & 3.131 & 1.821 & 2.957 & 0.085 & 22.904 \\
\hline
\end{tabular}

Fuente: elaboración propia a partir de datos de encuesta, 2015.

Un aspecto importante para obtener trabajo a la conclusión de sus estudios de licenciatura (variable respuesta), desde la perspectiva de los educandos fueron los temas que se impartieron en el área de idiomas, aquí los profesores fueron calificados con 8.3 puntos, se debe señalar que los estudiantes relacionan y dan importancias a los temas y la capacitación de los profesores de esta área. Este es un aspecto que las universidades debe- 
rán poner atención para satisfacer las necesidades de los estudiantes y del mercado laboral.

Con respecto a la cantidad de tiempo que invertirían en encontrar trabajo al egresar de la universidad, argumentaron que les llevaría en promedio 4.6 meses. Lo cual, no difiere mucho con respecto con los resultados presentados en un estudio realizado sobre los egresados de la Licenciatura en Turismo de la Universidad Autónoma de Hidalgo, en él se menciona que 73.7 por ciento de los hombres y 68.8 por ciento de las mujeres consiguieron su primer empleo en menos de 6 meses (Bezies et al., 2013). Los entrevistados piensan desempeñarse fundamentalmente en la administración pública (23.8 por ciento), en hoteles (21.3 por ciento), cruceros (17.2 por ciento), como microempresarios (16.4 por ciento), y en agencia de viajes, aeromoza y docencia (diez por ciento). Se encontró diferencia estadísticamente (Kruskal-Wallis $=9.332 ; \mathrm{p}=0.009)$ en el tipo de trabajo en el que piensan emplearse, los de la UIEP consideran la posibilidad de establecer un micro negocio (60 por ciento) relacionado con la actividad turística. En cambio, los de la BUAP mencionaron emplearse en la admiración pública (27.4 por ciento), en los hoteles (21.9 por ciento) y en los cruceros (20.5 por ciento por ciento) y los estudiantes de la UMAD buscarán trabajo en los hoteles (29.4 por ciento), en el gobierno (20.6 por ciento) y en los cruceros (17.6 por ciento). Tanto los hombres como las mujeres se emplearán en los hoteles, el gobierno y los cruceros, fundamentalmente.

Se puede decir que las perspectivas de empleo se consideran un poco apartada de la realidad; ya que Dema, Díaz y Chacaltana (2015) mencionan que la inserción laboral de los jóvenes es precaria, ya que tienen altas tasas de empleo informal o de empleo sin acceso a prestaciones laborales; también se puede mencionar el trabajo temporal, el trabajo a tiempo parcial, el trabajo temporal a través de agencia, las relaciones de empleo ambiguas y el trabajo autónomo son formas de trabajo comunes en el sector turístico (OIT, 2017). Esto sin tener en cuenta que por el contacto directo y la relación personal en la prestación de la mayoría de servicios de las ramas vinculadas al turismo una cantidad de empleos son de baja cualificación. Lo cierto es que existe desempleo — producto del modelo de desarrollo que ha llevado a una crisis económica mundial- y que la empleabilidad de los egresados en términos generales, no sea la adecuada, ya que no son empleados en actividades para la cual fueron capacitados, en ese sentido, los estudiantes entrevistados (75.4 por ciento) consideraron que existen posibilidades de encontrar trabajo en el lugar donde viven y sí no lo encuentran: la totalidad de cuestionados de la UMAD, 95.9 por ciento de la 
BUAP y 86.7 por ciento de la UIEP estarían dispuestos a cambiar de residencia para conseguirlo. Significa que los estudiantes de la UIEP tienen menor disposición a salir a trabajar fuera de su región. Se encontró que los hombres (100 por ciento) tienen mayor disposición que las mujeres (94.7 por ciento) a cambiar de residencia por cuestiones laborales. Si no encuentran empleo en lo que estudiaron, 67.6 por ciento de la UMAD, 65.8 por ciento de la BUAP y 80 por ciento de la UIEP estarían dispuestos a trabajar en actividades que no tienen que ver con la actividad turística, como sería la enseñanza de idiomas. Ello responde a la necesidad de laborar y no caer en el desempleo, ya que tienen la necesidad de obtener dinero para su manutención y recreación (Torres et al., 2017).

Se puede decir que las expectativas laborales de los entrevistados responden a la flexibilización del mercado de trabajo que busca contratar personal con menor calificación y cuando contratan personal calificado elevan los requisitos. Significa que los empresarios se aprovechan de la formación de los profesionistas, arguyendo que no tiene el perfil profesional al solicitar competencias profesiones que tienen alto volumen de demanda, perspectivas de crecimiento y profesiones emergentes que están surgiendo a escala global (Universidad Internacional de Andalucía-UIA, 2020). En turismo se puede hablar de que los empleos con mayor demanda son los de puestos base - camareros, cocineros, servicio de limpieza-, informador turístico/atención al cliente, recepcionista, y community manager, el cual, gestiona las redes sociales y la comunicación online, ajusta precios, realiza promociones y capta ventas (Aguado, 2016). Es por ello que el problema no es que no haya puestos de trabajo vacantes, sino que se arguye que los individuos no poseen las características y los conocimientos necesarios para cubrir estos puestos y que la universidad ofrece una escasa adaptación a los perfiles profesionales demandados por la sociedad.

Los estudiantes consideran que el encontrar empleo será difícil (37.7 por ciento) y regularmente difícil (46.7 por ciento). En la UMAD (50 por ciento), en la BUAP (43 por ciento) y en la UIEP (53.3 por ciento) comentaron que será regularmente difícil tener empleo y que será difícil o muy difícil en la BUAP ( 43.8 por ciento), en la UMAD (41.2 por ciento) y en la UIEP (33.3 por ciento). Tal vez este resultado se explique a que los estudiantes de UIEP van a buscar emprender su propio negocio. Estos resultados son muy semejantes a los reportados por el Centro de Opinión Pública de Laureate (2019) que mencionan que 45 por ciento de los encuestados consideró que obtener empleo fue difícil o muy difícil. Esta visión es propia de la vida cotidiana en la que se encuentra la población de nuestro país. 
El 61.8 por ciento de los encuestados de la UMAD, 79.5 por ciento de la BUAP y 66.7 por ciento UIEP mencionaron que el egresar de estas universidades será un factor importante para la búsqueda de empleo. Novella et al. (2018) en este sentido mencionan que también influye su preparación, los factores personales, la falta de experiencia previa, así como el género, el tipo de licenciatura, la situación económica de sus padres, y familiares, el ciclo económico y el lugar de residencia (Corrales y Rodríguez, 2003). Además, depende del contexto geográfico, social, de mercado, políticos, en donde se lleva la inserción, así como los factores personales como las estrategias de búsqueda de empleo, tiempo invertido en la búsqueda, expectativas profesionales y de salario. Asimismo, se debe tener en cuenta la formación profesional y la inserción y movilidad (ANECA, 2009).

Por lo que atañe al salario promedio que piensan ganar, este sería de $\$ 7,640.8$ mensuales, se encontró diferencia estadística $(F=4.373$; $\mathrm{p}=0.015)$ por universidad. Los de la UMAD, mensualmente esperaran ganar un salario mayor $(\$ 9,395.9)$ que los de la BUAP $(\$ 6,918.1)$ y los de la UIEP $(\$ 7,183.4)$. Ello se explica a que los alumnos de la UMAD, en términos generales invirtieron mayores recursos económicos en sus estudios. Pero, estadísticamente $(\mathrm{t}=4.413 ; \mathrm{p}=0.680)$ ganarían el mismo salario los hombres $(\$ 7,922)$ y las mujeres $(\$ 7,555.7)$. Se considera que un problema al que se enfrentan los recién egresados en turismo es su sueldo, el cual, ronda los tres y ocho mil pesos mensuales y dependerá de la empresa y el tipo de empleo que desempeñen (MEXTUDIA, 2021).

Los ingresos que obtienen los egresados de la licenciatura en turismo son relativamente bajos en comparación con la licenciatura en administración y gestión de empresas que se encontraba entre las regularmente pagadas $(\$ 12,350)$ - décimo noveno lugar-; y son bajos comparados con las licenciaturas mejor remuneradas, aquí destaca la Medicina $(\$ 17,889)$, Finanzas, banca y seguros $(\$ 17,050)$, Construcción e ingeniería civil $(\$ 14,848)$ (IMCO, 2021). También se debe tener presente que el nivel educativo está correlacionado con los salariales, significa que los ingresos parecen estar más relacionados con los puestos de trabajo que con el grado educativo, es decir, que están desarrollando un trabajo que requiere menos habilidades que su educación (OCDE, 2018) y se evalúa que existe un desajuste en los salarios y estos con el tiempo se corrigen, es por ello que las personas con un mayor nivel educativo son los que poseen mejores ingresos (Pérez, 2018). Entonces, son más altos los salarios de una persona con mayor nivel educativo comparados con los que perciben las personas que no asistieron a la universidad. 
Para los jóvenes de estas universidades el estudiar una licenciatura, significa mejorar su calidad de vida y en menor proporción: superarse, labrarse un futuro, independizarse de su familia y encontrar un mejor empleo; y consideran que si no hubieran tenido la oportunidad de estudiar serían empleados administrativos, tendrían un negocio propio y en menor proporción opinaron que se verían en cualquier trabajo o estarían en alguna rama empresarial y de negocios, también trabajarían en empresas de restauranteros o tendrían algún oficio. Esta percepción demuestra que entre los alumnos el cursar una licenciatura aún tiene importancia para mejorar sus condiciones de vida. En este contexto, un porcentaje superior al 86 por ciento piensa seguir estudiando después de concluir su licenciatura, 26.7 por ciento de la UMAD, 34.8 por ciento de la BUAP y 6.7 por ciento de la UIEP externaron que harían una maestría en ciencias. Para 23.3 por ciento de la UMAD, 16.7 por ciento de la BUAP y 3.3 por ciento de la UIEP desean hacer un diplomado y en menor porcentaje quieren estudiar otra licenciatura, tomarían algún curso, fundamentalmente. Esta decisión es para mejorar económicamente, ya que, entre más preparados estén existen mayores posibilidades de mejorar su calidad de vida. Aquí, toman relevancia los programas de capacitación, ya que su finalidad es contribuir a disminuir el desempleo considerando que entre mayor tiempo este sin laborar sus posibilidades de conseguir una contratación será menor (Padilla, 2017).

\section{Conclusiones}

La oferta de la licenciatura en turismo de las universidades de estudio son de reciente creación, no tienen más de diez años y ha adquirido mayor aceptación entre las mujeres. En el desarrollo de competencias profesionales la mayoría de estudiantes consideró que su formación académica será buena, y que serán competitivos laboralmente. En las competencias profesionales destacó la UMAD primordialmente en los idiomas ya que la totalidad habla el idioma inglés, aquí la UIEP presenta deficiencias. Los estudiantes de la BUAP y UMAD tienen una mejor precepción de las competencias desarrolladas tanto profesionales como transversales, en comparación con los de la UIEP que percibieron que las adquirieron en menor proporción. Pero ello no impidió para que, en términos generales, los estudiantes consideran que están bien preparados para incorporarse al mercado de trabajo tanto los de UIEP como UMAD y BUAP. Aquí las mujeres valoraron que serán más competitivas que los hombres.

Los estudiantes de las carreras de turismo de la UMAD, BUAP y UIEP tiene similar percepción de que al salir de la universidad sería posible ob- 
tener empleo en forma casi inmediatamente en su región de residencia, ya que consideran que están preparados y porque su carrera es muy demandada. Pero no tienen en cuenta que la inserción laboral de los jóvenes es difícil, por las altas tasas de desempleo que existen y si lo encuentran lo más seguro es que no tengan prestaciones laborales. Lo cierto es que no desarrollaron a plenitud las competencias profesionales y transversales y ello puede ser un obstáculo para encontrar trabajo. Es de destacar que los de la UIEP buscarán autoemplearse y los de la BUAP y UMAD intentarán emplearse en la admiración pública o en el sector privado como: en hoteles y cruceros. Los estudiantes de la UMAD y BUAP en mayor proporción consideraron que sí no lo encuentran trabajo en su lugar de origen están dispuestos a cambiar de residencia para conseguirlo. Significa que los estudiantes de la UIEP tienen menor disposición a salir a trabajar fuera de su región.

Por las condiciones del país, lo cierto es que los egresados se enfrentarán a la dificultad de encontrar empleo y si lo obtienen, muchos de estos empleos serán en actividades inferiores a sus competencias, y con puestos inferiores y salarios reducidos. En este sentido, los estudiantes de la UMAD, pretenden ganar mejores ingresos que los de la BUAP y UIEP y entre hombres y mujeres perciben que obtendrán los mismos salarios. Estos se consideran bajos con respecto a la inversión realizada en su licenciatura y comparados con otras carreras que se imparten en el país, aunque se considera normal comparado con el salario promedio que ganan los egresados de las universidades en México y alto en comparación a las personas que no estudiaron. A pesar de la situación a la que se enfrentaran los estudiantes consideraron que el estudiar una licenciatura, significará mejorar su calidad de vida y la Universidad se sigue viendo como una opción para mejorar sus condiciones de vida.

Se concluye que el panorama para encontrar empleo será complicado, y tendrá importancia en encontrarlo factores personales y familiares, así como las estrategias de búsqueda de empleo, tiempo a invertir en la búsqueda, expectativas profesionales y de salario. Una limitante en la investigación fue la escasa matricula que existe en las universidades privadas y en la universidad intercultural, lo que reduce la muestra y dificulta la comparación entre estratos (semestres) dentro de las universidades y, también sería importa profundizar respecto a las condiciones socioeconómicas de los padres de los estudiantes. Una investigación a futuro sería dar seguimiento a los egresados de estas universidades para conocer si tienen trabajo y la calidad de este. 
Perspectivas laborales de estudiantes de turismo en universidades de Puebla, México / M.J. CONTRERAS et al.

\section{Agradecimientos}

Este trabajo forma parte del Proyecto: Análisis del impacto de la educación dirigida a la población indígena en la Sierra Nororiental de Puebla, que fue financiado por el Consejo Nacional de Ciencia y Tecnología (CONACYT).

\section{REFERENCIAS BIBLIOGRÁFICAS}

Aguado, Javier, 2016, Los trabajos más demandados en el sector del turismo, Economía, Hosteltur, Disponible en https:/www.hosteltur.com/comunidad/ nota/002911_los-trabajos-mas-demandados-en-el-sector-del-turismo.html Consultado el 09/09/2021.

Aguilar Aguilar, Miguel Ángel, Crisanto Campos, Oscar y Sánchez Daza, German, 2013, "Educación superior y desarrollo en América Latina: un vínculo en debate. ¿La necesaria mediación del mercado?”, en Martínez de Ita, María Eugenia, Julio Piñero, Fernando y Figueroa Delgado, Silvana Andrea (Coords.), El papel de la universidad en el desarrollo, Benemérita Universidad Autónoma de Puebla y Universidad Nacional del Centro de la Provincia de Buenos Aires, pp. 23-44.

América Economía, 2015, América Economía. Ranking de universidades de México, Disponible en http://rankings.americaeconomia.com/mejores-universidades-mexico-2015/ Consultado el 23/04/2015.

ANECA, 2009, "Los procesos de inserción laboral de los titulados universitarios en España", en Factores de facilitación y de obstaculización. Madrid: ANECA.

ANUIES, 2014, Anuario Educación Superior de la población escolar en la educación superior. Técnico superior y licenciatura, ciclo escolar 2013- 2014. México; ANUIES. Disponible en www.anuies.mx/iinformacion-y-servicios/informacion-estadistica-de-educacion-superior/anuario-estadistico-de-educacion-superior Consultado el 15/05/2015.

ANUIES, 2019, Anuario Educación Superior de la población escolar en la educación superior. Técnico superior y licenciatura, ciclo escolar 2018- 2019. México, Disponible en http://www.anuies.mx/informacion-y-servicios/informacion-estadistica-de-educacion-superior/anuario-estadistico-de-educacion-superior Consultado el 05/09/2021.

Benavides Abanto, Martín, 2004, "Educación y estructura social en el Perú. Un estudio acerca del acceso a la educación superior y la movilidad intergeneracional en una muestra de trabajadores urbanos", en ¿Es posible mejorar la educación peruana? Evidencias y posibilidades, Didi de Arteta S. A., Lima.

Bezies Cruz, Patricia, Pérez Enzastiga, José Antonio, González Mociños, Nancy Herrera Baez, Carlos, Elizalde Lora, Leticia, Olvera Larios, Brenda Ivonne, López Cruz, María De Los Ángeles, Enciso Islas, Alberta, Ordaz Monroy, Adriana Anabel, Ávila Gallardo, Wendolyn, Gayosso Arias, Ignacio Nathanael y Zacatenco Luna, Misael, 2013, Resultados del estudio de egresados de la licenciatura en turismo, Universidad Autónoma del Estado de Hidalgo. Disponible en https://www. 
uaeh.edu.mx/adminyserv/dir_generales/evaluacion/spitel/seguimiento_de_egresados/54_turismo_2013_pdf Consultado el 06/09/2021.

BUAP, 2021, Oferta educativa. Licenciatura en Administración Turística. Facultad de Administración, Benemérita Universidad Autónoma de Puebla, Puebla, Disponible en https://admision.buap.mx/sites/default/files/Planes\%20de\%20Estudio/2020/Econ\%C3\%B3mico\%20Administrativas/Lic.\%20en\%20Administraci\%C3\%B3n\%20Tur\%C3\%ADstica.pdf Consultado el 13/09/2021.

Burgos Flores, Benjamín y López Montes, Karla, 2010, "La situación del mercado laboral de profesionistas” en Educación Superior, vol. 39 (4), Núm. 156, p. 19-33.

Capsada Munsech, Queralt, 2017, “Overeducation: Concept, theories, and empirical evidence", en Sociology Compass, vol. 11, núm. 10 https://doi.org/10.1111/ soc4.12518 p.01-17.

Carlson, Beverley, 2002b, Educación y mercado de trabajo en América Latina: ¿qué nos dicen las cifras, Serie de Desarrollo Productivo, CEPAL, Núm. 77, Santiago de Chile.

Centro de Opinión Pública de Laureate, 2017, Encuesta nacional de egresados. Reporte de resultados, Universidad del Valle de México, Disponible en https:// profesionistas.org.mx/wp-content/uploads/2017/06/Encuesta-Nacional-de-Egresados.pdf Consultado el 10/09/2021.

Centro de Opinión Pública de Laureate, 2019, Encuesta nacional de egresados. Un estudio para contribuir a la mejora de la Educación Superior en México, Universidad del Valle de México, Disponible en https://www.observatoriolaboral.gob. $\mathrm{mx} /$ static/estudios-publicaciones/ENE-2019digital.pdf Consultado el 12/09/2021.

Chossudovsky, Michel, 2007, "La mano de obra barata en la economía global", en Global Research, Deslinde.org.co 31 Mayo, Disponible en www.globalresearch.ca/la-mano-de-obra-bara ta-en-la-econom-a-global/5828 Consultado el 05/06/2015.

Comisión Económica para América Latina y El Caribe (CEPAL)/Organización Internacional del Trabajo (OIT), 2019, "El futuro del trabajo en América Latina y el Caribe: antiguas y nuevas formas de empleo y los desafíos para la regulación laboral”, Coyuntura Laboral en América Latina y el Caribe, Núm. 20 (LC/ TS.2019/31), Santiago de Chile.

Corrales Herrero, Helena y Rodríguez Prado, Beatriz, 2003, "La transición del sistema educativo al mercado laboral Análisis de los factores y determinantes del primer desempleo", en $8^{\circ}$ Congreso de Economía Regional de Castilla y León, 1 , Consejería de Economía y Hacienda, Valladolid.

Danvila del Valle, Ignacio, Sastre Castillo, Miguel Ángel y Marroquín Tovar, Edmundo, 2013, "The importance of professional competencies in the development of emotional intelligence", en Internacional de Organizaciones, núm. 10, p. 156179. 
Dema Rey, Guillermo, Díaz Noziglia, Juan José y Chacaltana Janampa, Juan, 2015, ¿Qué sabemos sobre los programas y políticas de primer empleo en América Latina?, OIT, Lima.

Domínguez Lugo, Alma Jovita, Silva Ávila, Alicia Elena, Castorena Peña, Abraham, Barrera Moreno, Mario Alberto y Ramírez González, Dora Isabel, 2017, "Investigación sobre las oportunidades de empleo para los profesionistas recién egresados utilizando BSC", en Iberoamericana para la Investigación y el Desarrollo Educativo, Vol. 8, Núm. 15. DOI: 10.23913/ride.v8i15.293

Expansión, 2020, Los jóvenes no trabajan en lo que estudiaron... y en las empresas falta talento, Disponible en https://expansion.mx/carrera/2020/01/30/jovenes-no-trabajan-estudiaron-empresas-falta-talento, Consultado el 09/09/2021.

García, Luis Fernando y Cruz, Moritz, 2017, "Desempleo en América Latina: ¿flexibilidad laboral o acumulación de capital?", en Problemas del Desarrollo, vol. 189, núm. 48, http://probdes.iiec.unam.mx 33, p. 33-56.

Gobernado Arribas, Rafael, 2007, "La sobreeducación en España: estudio descriptivo y revisión crítica del concepto", en Papers, núm. 86, p. 11-31.

Gómez Aguilar, Roberto, 1979, Introducción al muestreo, Disertación de Maestría en Ciencias en Estadística, Centro Estadística y Cálculo, Colegio Postgraduados, México.

Gómez Urrutia, Verónica y Royo Urrizola, Paulina, 2015, "Nuevas subjetividades y proyecto de vida: jóvenes universitarios de la VII región del Maule", en Estudios Sociales, Chile, núm. 53, p. 90-101.

Hernández, Roberto, Fernández, Carlos y Baptista, María del Pilar, 2014, Metodología de la investigación. McGraw-Hill / Interamericana Editores.

Hosmer, David W.; Lemeshow, S. Tanley and Sturdivant Rodney X., 2013, Applied logistic regression. John Wiley and Sons.

IMCO, 2021, Compara Carreras Una herramienta sobre las consecuencias económicas de escoger una carrera, México; Instituto Mexicano para la Competitividad, Disponible en https://imco.org.mx/comparacarreras/las-10-mas/porcentaje-desempleados/2021/1. Consultado el 03/09/2021.

INEGI, 2016, Panorama Sociodemográfico de Puebla 2015. Instituto Nacional de Estadística y Geografía, México. Disponible en https://www.inegi.org.mx/contenido/productos/prod_serv/contenidos/espanol/bvinegi/productos/nueva_estruc/ inter_censal/panorama/702825082314.pdf Consultado el 01/09/2021.

Instituto Nacional de Estadística y Geografía, 2017, Anuario estadístico y geográfico de Puebla 2017. Instituto Nacional de Estadística y Geografía, México. Disponible en https://www.datatur.sectur.gob.mx/ITxEF Docs/PUE_ANUARIO_PDF.pdf, Consultado el 01/09/2021. Consultado el 01/09/2021.

Linarez Placencia, Gildardo, 2014, "Calidad en las instituciones de educación superior en México: ¿utopía o desafío?”, en Iberoamericana para la Investigación y el Desarrollo Educativo, núm.12. 
López Pina, Pilar y García Hernández, José Antonio, 1998, “Acceso laboral y formación turística”, en Cuadernos de Turismo, Murcia, núm. 1, p. 91-98.

Mateos Cortés, Laura Selene y Dietz, Gunther, 2016, "Universidades interculturales en México balance crítico de la primera década", en RMIE, vol. 21, núm. 70, p. 683-690.

Mendoza Ontiveros, Martha Marivel y Chapulín Carrillo, Jazmín de Verano, 2015, "Turismo, trabajo femenino y empoderamiento de las mujeres en Bahías de Huatulco, Oaxaca - México", en Estudios y Perspectivas en Turismo, vol. 24, p. 316335 .

Mendoza, Marcela, 1994, "Técnicas de observación directa para estudiar interacciones sociales infantiles entre los Toba”, en RUNA, vol. 21, núm. 1, p. 241-262.

MEXTUDIA, 2021, Turismo, Disponible en https://mextudia.com/carreras/turismo/ Consultado el 17/09/2021.

Morales Sandoval, Berenice y Castro Lugo, David, 2015, "La flexibilidad del mercado de trabajo: causas, conceptualización y expresión", en Equilibrio Económico, vol. 11, núm. 39, p. 07-40.

Murillo García, Favio y Montaño Ulloa, Paulina Yolanda, 2018, "Condiciones laborales de egresados de Instituciones de Educación Superior en México", en Investigación Educativa, vol. 20, núm. 3. DOI: https://doi.org/10.24320/redie.2018.20.3.1644.

Novella, Rafael, Repetto, Andrea, Robino, Carolina y Rucci, Graciana, 2018, Millennials en América Latina y el Caribe: ¿trabajar o estudiar?, Banco Interamericano de Desarrollo. División de Mercados Laborales.

OCDE, 2018, Education at a Glance 2018: OECD Indicators, OECD Publishing, Paris, en http://dx.doi.org/10.1787/eag-2018. Consultado el 08/9/2021.

OCDE, 2019, Higher Education in Mexico: Labour Market Relevance and Outcomes, Higher Education, OECD Publishing, Paris, en: https://doi. org/10.1787/9789264309432. Consultado el 28/08/2021.

OCDE, 2020, Education at a Glance 2020: OECD Indicators, OECD Publishing, Paris, en https://doi.org/10.1787/69096873. Consultado el 08/09/2021.

OIT, 2014, Panorama Laboral 2014. Lima: Organización Internacional del Trabajo / Oficina Regional para América Latina y el Caribe.

OIT, 2015, Panorama Laboral 2015. Lima: Organización Internacional del Trabajo / Oficina Regional para América Latina y el Caribe.

OIT, 2017, Pautas de la OIT sobre trabajo decente y turismo socialmente responsable. Oficina Internacional del Trabajo, Departamento de Políticas Sectoriales. Ginebra, OIT.

OIT, 2020, Panorama Laboral 2020. América Latina y el Caribe. Lima, Organización Internacional del Trabajo / Oficina Regional para América Latina y el Caribe. 
OIT, 2021, Nota técnica. Hacia una recuperación sostenible del empleo en el sector del turismo en América Latina y el Caribe. OIT, Serie Panorama Laboral en América Latina y el Caribe.

Organización Mundial del Turismo, 2019, Desarrollo del talento humano para la mejora de la competitividad de los destinos. El caso de Perú, Organización Mundial del Turismo, Madrid, España. DOI: 10.18111/9789284420766

Organización Mundial del Turismo, 2021, Panorama del turismo internacional, Edición 2020, OMT, Madrid,pp. 23. DOI: https://doi.org/10.18111/9789284422746

Organización Mundial del Turismo-OMT, 2015, Panorama OMT del turismo internacional, Madrid, Disponible en https://www.publication /panorama-omt-del-turismo-internacional-edicion-2015. Consultado el 04/05/2015.

Padilla Ruezga, Adrián, 2017, La inserción al mercado laboral de los egresados de los programas de licenciaturas administrativas de Centro Universitario de Los Altos, Tesis para obtener el grado de maestro en Administración de Negocios, Centro Universitarios de los Altos Universidad de Guadalajara.

Pérez Benítez, Jessica Ivonne, 2018, Inserción laboral de jóvenes universitarios por el régimen jurídico y el prestigio de la IES de procedencia en la Zona Metropolitana de la Ciudad de México, 2010. Tesis de maestría en Población y desarrollo Facultad Latinoamericana de Ciencias Sociales, México.

Pregibon, Daryl, 1981, "Logistic regression diagnostics", en The Annals of Statistics, vol. 9, núm. 4, p. 705-724. https://projecteuclid.org/download/pdf_1/euclid. $\operatorname{aos} / 1176345513$

Presidencia de la República, 2015, Tercer informe de Gobierno 2014-2015. México; Gobierno de la República, Presidencia de los Estados Unidos Mexicanos, Disponible en http://www.presidencia.gob.mx/tercerinforme/ Consultado el 17/06/2014.

Rahona López, Martha, 2008, "Un análisis del desajuste educativo en el primer empleo de los jóvenes”, en Principios-Estudios de Economía Política, núm. 11, p. 45-67.

Ramírez Guerrero, Ana María, 2018, Mejora continua de un programa educativo a través de la satisfacción de los consumidores. Caso: licenciatura en turismo del CUCEA, Tesis de maestro en Dirección de mercadotecnia del Centro Universitario en Ciencias Administrativas de la Universidad de Guadalajara.

Regalado Pezúa, Otto y Oré Butler, Claudia, 2009, La Educación superior en turismo y las necesidades reales del mercado, Universidad ESAN, Serie Documentos de Trabajo, Núm. 23, Lima.

Rivas García, Rosa María, Cardoso Espinosa, Edgar Oliver y Cortés Ruiz, Jesica Alheli, 2019, "Propuesta de las competencias profesionales en turismo desde el enfoque del empleador", en Iberoamericana para la Investigación y el Desarrollo Educativo, vol. 9, núm. 18. DOI: https://doi.org/10.23913/ride.v9i18.418

Rodríguez Rey, Laura y Moreno, Eliana M., 2017, Estrategias para disminuir el impacto del desempleo en la salud mental. El papel de la motivación y la autoefi- 
cacia en la orientación laboral, Editorial Departamento de Psicología. Facultad de Ciencias de la Educación. Universidad de Córdoba, Impreso en Córdoba, España.

Rubio-Hernández, Lisy y Salgado Vega, María del Carmen, 2014, “Características del proceso de transición al mercado laboral. Caso de los egresados de los licenciados en economía del Estado de México, 2000-2010", en Investigación Educativa, núm. 19, p.28-59.

Sáez-López, José-Manuel, Domínguez-Garrido, María-Concepción, Medina-Domínguez, María-del-Castañar, Monroy, Fuensanta y González-Fernández, Raúl, 2021, "The competences from the perception and practice of university students", en Social Sciences, vol. 10, núm. 34. DOI: https://doi.org/10.3390/socsci10020034

Salmi, Jamil, 2009, El desafio de crear universidades de rango mundial, Banco Mundial en coedición con Mayol Ediciones, Colombia.

Sánchez Sánchez, Nuria y Fernández Puente, Adolfo C., 2014, “Desajuste educacional y de competencias: efectos diferenciales sobre la satisfacción laboral. Un estudio aplicado al mercado de trabajo español", en Estudios de Economía, vol. 41, núm. 2, p. 261-281.

SECTUR, 2021, Resultados de la Actividad Turística. Enero 2021. DataTur. Unidad de Asuntos y Cooperación Internacionales, Secretaría de Turismo. Disponible en https://www.datatur.sectur.gob.mx/SitePages/versionesRAT.aspx Consultado el 17/09/2021.

SEP, 2020, Principales cifras del Sistema Educativo Nacional. 2019-2020. México: Dirección General de Planeación y Estadística Educativa, Secretaría de Educación Pública, Disponible en https://www.planeacion.sep.gob.mx/Doc/estadistica_e_indicadores/principales_cifras/principales_cifras_2019_2020_bolsillo.pdf Consultado el 28/08/2021.

Silas Casillas, Juan Carlos, 2005, "Realidades y tendencias en la educación superior privada mexicana”, en Perfiles educativos, vol. 27, núm. 109-110, p. 07-37.

Torres Crespo; María Rosa, 2020, "Estudio sobre la actitud y las expectativas de los estudiantes del grado en turismo de la Universidad de Almería", en Know and Share Psychology, vol. 1, núm. 2, p. 63-75.

Torres López, Teresa Margarita, Acosta Fernández, Martín y Aguilera Velasco, María de los Ángeles, 2017, "Preparación para el trabajo: Representaciones sociales del empleo y desempleo de jóvenes mexicanos sin trayectoria laboral", en Educación y Desarrollo Social, vol. 11, núm. 1, p. 85-101. DOI: http://dx.doi. org/10.18359/reds. 1867

Tribe, John, 1999, Economía del ocio y el turismo, Editorial Síntesis. S. A.

UIEP, s/f, UIEP: Plan de estudios. Turismo alternativo, Puebla, Universidad Intercultural del Estado de Puebla y Secretaría de Educación Pública, Disponible en file://C:/Users/DRJped/Down loads/uiep_-fracc-i-art.-21-plan-de-estudios_turismo-a-2014---uiep\%20(2) .pdf Consultado el 09/09/2021. 
UMAD, 2021, UMAD: Oferta educativa. Licenciatura en Negocios Turísticos, Puebla, Área de Negocios de la Universidad Madero, Disponible en https://umad. edu.mx/negocios-turisticos/ Consultado el14/09/2021.

Universidad Internacional de Andalucía, 2020, Estudio de perfiles profesionales y habilidades asociadas, Observatorio UNIA de Perfiles Profesionales, Universidad Internacional de Andalucía, Disponible en https://www.unia.es/images/observatorios/perfiles-profesionales/2020_Observatorio_Perfiles_completo.pdf Consultado el 06/09/2021.

Valenti Negrini, Giovanna y Becerril Posadas, Gabriela, 2007, “¿Existe subutilización detrás de la sobreeducación de los profesionistas en México?”, en Trabajo, núm. 5, p. 37-59.

Vargas Moreno, Paola, 2014, "Educación Superior Intercultural en Disputa. Trayectorias de la Universidad Intercultural Amawtay Wasi", en Polis, vol. 13, núm. 38, p. 269-300.

Villalobos Monroy, Guadalupe y Pedroza Flores, René, 2009, "Perspectiva de la teoría del capital humano acerca de la relación entre educación y desarrollo económico", en Tiempo de Educar, vol. 10, núm. 20, p. 273-306.

World Tourism Organization-UNWTO, 2020, "Barometer", en Organización Mundial del Turismo, vol. 18, núm. 4, p. 25.

World Travel and Tourism Council-WTTC, 2021, Global economic impact and trends 2021. Global. About WTTC and Oxford Economics, Disponible en https://wttc.org/Portals/0/Documents/Reports/2021/Global\%20Economic\%20Impact $\% 20$ and $\% 20$ Trends\%202021.pdf?ver=2021-07-01-114957-177 Consultado el 31/08/2021.

\section{RESUMEN CURRICULAR DE LOS AUTORES}

\section{María José Contreras López}

Maestra en Ciencias en Estrategias para el Desarrollo Agrícola Regional por el Colegio de Postgraduados y Licenciada en Administración Turística por la Universidad Madero. A la fecha ha publicado diversos artículos y capítulos de libro. Ha realizado dos tesis, una de licenciatura y otra de maestría, ambas relacionadas con el turismo y el desarrollo rural. En docencia ha impartido asignaturas relacionadas con el área de turismo a estudiantes de licenciatura en Administración Turística, en el Complejo Regional Mixteca, ubicado en el municipio de Atlixco de la Benemérita Universidad Autónoma de Puebla. Su línea de investigación es el turismo rural y actualmente se desempeña como Investigadora Auxiliar Adjunta, 
asignada al posgrado en Estrategias para el Desarrollo Agrícola Regional del Campus Puebla.

Dirección electrónica: majo.contreras1@hotmail.com

Registro ORCID: https://orcid.org/0000-0002-3260-9246

\section{José Pedro Juárez Sánchez}

Obtuvo su doctorado en Geografía y un Diploma de Estudios Avanzados en Planificación Territorial y Desarrollo Regional por la Universidad de Barcelona, España, es Maestro en Ciencias por el Colegio de Postgraduados y es Licenciado en Administración Pública por la Benemérita Universidad Autónoma de Puebla. Sus líneas de investigación son: turismo rural en regiones indígenas, política agrícola, migración, pobreza, vivienda, alimentación en regiones rurales y evaluación de programas de desarrollo. Es Profesor de la maestría de Paisaje y Turismo Rural y en la maestría y doctorado en Estrategias para el Desarrollo Agrícola Regional (PROEDAR) ambas pertenecen al Programa Nacional de Postgrados de Calidad del CONACYT. Ha dirigido y asesorado un importe número de tesis de maestría y doctorado, fundamentalmente del PNPC. También he participado en una importante cantidad de congresos internacionales y nacionales, en cursos de capacitación y en la dirección de programas de desarrollo agrícola. Coordinó la línea de investigación de Estudios Regionales del Colegios de Postgraduados. Actualmente es miembro del Comité de posgrado en Estrategias para el Desarrollo Agrícola Regional. Pertenece a la Academia Mexicana de Ciencias. Es miembro del Sistema Nacional de Investigadores Nivel II.

Dirección electrónica: pjuarez@colpos.mx

Registro ORCID: https://orcid.org/0000-0001-8417-1752

\section{Benito Ramirez Valverde}

$\mathrm{Ph}$. D. en Estudios Latinoamericanos, Tulane University. Estados Unidos. Maestría en Estudios Latinoamericanos Tulane University. Nueva Orleans, Estados Unidos. Maestría en Ciencias en Estadística, Colegio de Postgraduados; Ingeniero Agrónomo especialista en Fitotecnia, Universidad Autónoma Chapingo. Sus líneas de investigaciones son: Desarrollo rural, pobreza rural y evaluación de programas de desarrollo, en el Colegio de Postgraduados. Ha sido Director del Campus Puebla del Colegio de Postgraduados (2002-2009). Es Director de la revista Agricultura, Sociedad y Desarrollo, indexada en el Índice de Revistas Mexicanas de Investigación del Consejo Nacional de Ciencia y Tecnología (CONACYT). Profesor de 
Perspectivas laborales de estudiantes de turismo en universidades de Puebla, México / M.J. CONTRERAS et al.

la Maestría y Doctorado en Estrategias para el Desarrollo Agrícola Regional. Ha impartido cursos a nivel licenciatura y posgrado en diversas instituciones del país y a nivel internacional ha participado en cursos en Guatemala, Nicaragua y Brasil. Entre las distinciones que el Dr. Ramírez ha recibido se encuentran: El Premio Estatal de Ciencia y Tecnología 2004; y el Premio Estatal al Mérito Agropecuario 2007. Pertenece a la Academia Mexicana de Ciencias. Es miembro del Sistema Nacional de Investigadores Nivel II.

Dirección electrónica: bramirez@colpos.mx

Registro ORCID: http://orcid.org/0000-0003-2482-5667 